\title{
Impacto de factores socioeconómicos en la mortalidad por suicidios en Colombia, 2000-2013*
}

\section{Impact of Socioeconomic Factors on Suicide Mortality in Colombia, 2000-2013}

\section{Impacto de fatores socioeconómicos na mortalidade por suicídios na Colômbia, 2000-2013}

Fecha de recepción: 26-01-2017 Fecha de aceptación: 17-04-2017 Disponible en línea: 30-05-2017 doi:10.11144/Javeriana.rgps16-33.ifsm

Cómo citar este artículo:

Dávila-Cervantes CA, Pardo-Montaño AM . Impacto de factores socioeconómicos en la mortalidad por suicidios en Colombia, 2000-2013. Rev Gerenc Polít Salud. 2017; 16 (33) : 36-51. https://doi.org/10.11144/ Javeriana.rgps16-33.ifsm

\footnotetext{
* Artículo de investigación.

** Autor principal. Doctor en Estudios de Población, profesor investigador de la Facultad Latinoamericana de Ciencias Sociales sede México (Flacso). Correo electrónico: claudio.davila@flacso.edu.mx

*** Autora de correspondencia. Doctora en Geografía, investigadora asociada del Instituto de Geografía de la Universidad Nacional Autónoma de México, Universidad Nacional Autónoma de México, Circuito de la Investigación Científica, Ciudad Universitaria, 04510, Coyoacán, México. Correo electrónico: apardo@igg.unam.mx
} 


\section{Resumen}

El objetivo fue analizar la tendencia, el impacto y los factores socioeconómicos asociados con la mortalidad por suicidios en Colombia entre el 2000 y el 2013, por sexo, grupos de edad y estratos del índice de necesidades básicas insatisfechas (INB). Se calcularon tasas estandarizadas de mortalidad, años de vida perdidos (AVP) y un análisis de regresión múltiple binomial negativa. La mortalidad por suicidios disminuyó en Colombia entre el 2000 y el 2013. El estrato medio INB tuvo la mayor mortalidad por suicidios; el estrato muy bajo presentó la mayor disminución; y el estrato muy alto tuvo la menor mortalidad. La desigualdad del ingreso se asoció positivamente con el riesgo de fallecer por suicidio, al igual que la tasa de desempleo y la pobreza tienen una relación negativa con la mortalidad de suicidios. Además de los factores de riesgo individuales del suicidio, las condiciones socioeconómicas del entorno deben ser considerados factores de riesgo de suicidio en Colombia.

Palabras clave: suicidio; mortalidad; Colombia; esperanza de vida; pobreza; factores socioeconómicos

\section{Abstract}

This aarticle analyzes the trend, the impact, and the socioeconomic factors associated with suicide mortality in Colombia between 2000 and 2013, according to gender, age groups, and strata of the unsatisfied basic needs index (AVP). We calculated the standardized mortality, lost years of life (AVP), and carried out a negative binomial multiple regression analysis. Mortality from suicides decreased in Colombia between 2000 and 2013. The middle-income stratum INB had the highest mortality from suicides; the very low income stratum showed the greatest decrease; and the very high income stratum had the lowest mortality. Income inequality was positively associated with the risk of dying from suicide, just as the unemployment rate and poverty are negatively related to suicide mortality. In addition to individual risk factors for suicide, socioeconomic conditions in the environment should be considered risk factors for suicide in Colombia.

Keywords: suicide; mortality; Colombia; life expectancy; poverty; socioeconomic factors

O objetivo foi analisar a tendência, o impacto e os fatores socioeconómicos associados a mortalidade por suicídio na Colômbia entre 2000 e 2013, por sexo, camadas de idade e nível de rendas do índice de necessidades básicas insatisfeitas (INB). Calcularam-se taxas padronizadas de mortalidade, anos de vida perdidos (AVP) e análise de regressão múltipla binomial negativa. A mortalidade por suicídio diminuiu na Colômbia entre 2000 e 2013. O nível meio de rendas INB teve a maior mortalidade por suicídio; o nível de rendas muito baixas apresentou a maior diminuição; e o nível de muito altas rendas teve a menor mortalidade. A desigualdade do ingresso foi associada positivamente com o risco de falecer por suicídio, igual que a taxa de desemprego e a pobreza têm relação negativa com a mortalidade de suicídios. Além dos fatores de risco individuais do suicídio, as condições socioeconómicas do entorno devem se considerar fatores de risco de suicídio na Colômbia.

Palavras chave: suicídio; mortalidade; colômbia; expectativa de vida; pobreza; fatores socioeconômicos 


\section{Introducción}

Los suicidios son una de las principales causas de muerte a escala mundial y uno de los principales problemas de salud pública, ya que representan cerca de la mitad de muertes violentas para hombres y más del $70 \%$ para mujeres (1-4). En el año 2000 se reportaron aproximadamente 815000 suicidios en el mundo, con una tasa de 14.5 suicidios por 100000 habitantes (5); mientras que en el 2012 se registraron 804000 casos, con una tasa de 11.4 suicidios (15.0 entre hombres y 8.0 entre mujeres) (1). Se estima que el suicidio es la segunda causa principal de muerte a escala mundial en el grupo de quince a veintinueve años de edad; y entre los adultos de 30 a 49 años es la quinta (1.6). Los suicidios producen una gran pérdida social, emocional y económica en las personas que están alrededor del suicida, además de elevados costos sociales y económicos para la sociedad (7).

De acuerdo con la Organización Mundial de la Salud (OMS), tres cuartas partes de los suicidios ocurren en países de ingresos bajos y medios, lo que implica que suceden en contextos de escasos recursos y servicios donde se dificulta que se pueda llevar a cabo una identificación temprana de los factores de riesgo que pueden desencadenar el suicidio (1). El principal factor de riesgo del suicidio es un intento previo; $y$ se ha estimado que por cada suicidio consumado se producen más de veinte intentos $(3,6,8)$, los cuales acarrean una enorme carga social y económica, debido a que en muchas ocasiones se traducen en lesiones, hospitalizaciones, traumas emocionales y, en ciertos casos, en discapacidades a largo plazo $(1,3,6,9)$.

Dada la importancia del fenómeno, se ha buscado su comprensión a partir de la identificación de los factores económicos, sociales y culturales que se relacionan con su incidencia (10-14). Desde el punto de vista socioeconómico, se ha observado que la incidencia de suicidios tiende a incrementarse en épocas de crisis económicas, durante las cuales el desempleo, la pobreza y la desigualdad aumentan (15-16). Sin embargo, en Latinoamérica existen pocas investigaciones que hayan explorado la asociación entre el suicidio y las características socioeconómicas de la población, como la desigualdad, la pobreza o el desempleo (2).

En Latinoamérica ocurren más de 60000 defunciones por suicidio anualmente $(1,17)$ y en los últimos años se ha presentado un constante incremento de la mortalidad por esta causa $(17,18)$. Si bien en Colombia las tasas de mortalidad por suicidios son relativamente bajas, si se compara con otros países de la región (19), a finales de los años noventa y principios de la década pasada se observó un aumento importante de la mortalidad por esta causa, después de un periodo relativamente estable entre 1973 y 1995 (19-20). Por su lado, en el país, en los últimos años han disminuido la desigualdad, la pobreza y el desempleo (21). Ante esta situación, resulta importante analizar la posible asociación entre dichos indicadores y el comportamiento de la mortalidad por suicidios en Colombia.

Se ha estudiado que los factores de riesgo individuales por si solos no explican diferencias en la mortalidad por suicidios; se sugiere, entonces, que diferentes características del entorno social y económico en un periodo específico tienen efectos significativos en la mortalidad por suicidios (2). Dada esta situación y que el suicidio es un grave problema de salud pública en Colombia, el objetivo principal de este estudio es analizar la tendencia, el impacto y los factores socioeconómicos asociados con la mortalidad por suicidios en Colombia entre el 2000 y el 2013, por sexo y grupos de edad, y realizando una estratificación por medio del índice de necesidades básicas insatisfechas (INB). Para cumplir con 
dicho objetivo, se utilizan las tasas estandarizadas de mortalidad, los años de vida perdidos (AVP) en menores de cien años de edad y un análisis de regresión múltiple, por medio de modelos de regresión binomial negativa, para analizar los factores socioeconómicos asociados con la incidencia del suicidio.

\section{Material y métodos}

Se realizó un estudio mixto, con un diseño ecológico, transversal y con datos tipo panel. La información se obtuvo de las estadísticas vitales de mortalidad y de las series de indicadores sociales y económicos provenientes del Departamento Administrativo Nacional de Estadística (DANE) de Colombia. Los datos de defunciones se obtuvieron para el periodo 2000-2013, a escala departamental, por sexo y grupos quinquenales de edad. La población a mitad de año se obtuvo de las estimaciones de población del DANE. La información sobre el INB, el desempleo, el índice de Gini y la incidencia de la pobreza se obtuvo de las series históricas del DANE sobre dichos temas para los años y departamentos disponibles ${ }^{1}$. Se adoptó la definición de suicidios de la Clasificación Internacional de Enfermedades (CIE-10), en la cual estos aparecen bajo el rubro causas externas de mortalidad (códigos X60-X84; Y870). Se excluyeron del análisis las defunciones por suicidios en cuyo registro no constaban el sexo (178 casos, lo que equivale a $0,6 \%$ del total de suicidios registrados); se realizó un prorrateo para distribuir las muertes de las edades no especificadas entre el resto de los grupos de edad, a efectos de minimizar la pérdida de información.

1 Cabe señalar que la información puesta a disposición por el DANE para estas variables abarca solamente el periodo 2002 a 2013 e incluye información solamente para veinticuatro departamentos incluidos en la Encuesta Continua de Hogares y la Gran Encuesta Integrada de Hogares del DANE.
Se calcularon las tasas estandarizadas de mortalidad por suicidios, a escala departamental, por sexo y grupos quinquenales de edad, para el periodo de estudio, utilizando como estándar la población nacional del 2013. De igual forma, se calcularon los años de vida perdidos (AVP) propuestos por Arriaga (22), para estimar cuántos años de vida, en promedio, se perdieron entre el 2000 y el 2013 por suicidios de personas menores de cien años de edad (22). Se ocupó el supuesto de mortalidad nula entre cero y cien años de edad, el cual tiene la ventaja de que al comparar la mortalidad observada con la mortalidad hipotética nula, los resultados quedan estandarizados y se puedan realizar comparaciones históricas o entre poblaciones. Se seleccionó este rango etario debido a que, para este indicador, se recomienda tomar la edad más alta posible (22). Esta técnica permite relacionar los cambios en la mortalidad por suicidios con la esperanza de vida temporaria entre las edades analizadas (23) y, a partir de ello, dar cuenta del impacto de la mortalidad por suicidios en la salud de la población.

Para analizar la posible relación entre la mortalidad por suicidios y la pobreza, y con base en ello aportar mayor evidencia en torno a este debate, se utilizó el INB. Este índice se utiliza para estimar la magnitud de la pobreza en relación con la población, partiendo del supuesto de que las personas que habitan en viviendas con necesidades básicas insatisfechas o en situación de miseria se encuentran en las mismas condiciones de su respectiva vivienda (24). En este sentido, es importante mencionar que, en Colombia, desde finales de los años ochenta, la pobreza ha sido medida con la metodología de necesidades básicas insatisfechas (25). Se calcula por medio de cinco indicadores simples de las viviendas clasificadas como: inadecuadas, con hacinamiento crítico, con servicios inadecuados, con alta dependencia económica o con niños en edad escolar que no asisten a la escuela (24). 
El índice se mide en valores porcentuales, donde $100 \%$ indica el número más alto de necesidades insatisfechas de la población; este indicador osciló entre $9.2 \%$ (Bogotá) y $79.2 \%$ (Chocó) en el 2011, año para el cual se tiene la medición del índice. A partir de este indicador se hizo, por tanto, una estratificación de distribución por quintiles con el propósito de obtener cinco estratos de pobreza: muy bajo, bajo, medio, alto y muy alto (tabla 1 ).

T Abla 1. Estratificación socioeconómica a nivel departamental. Colombia, 2011

\begin{tabular}{|l|l|l|}
\hline \multicolumn{1}{|c|}{ NBI (\#) } & $\begin{array}{c}\text { Rango del percentil } \\
(\%)\end{array}$ & \multicolumn{1}{|c|}{ Departamentos } \\
\hline Muy Alto (6) & $54.9-79.2$ & Chocó, Córdoba, Guainía, La Guajira, Sucre, Vichada \\
\hline Alto (7) & $42.6-54.8$ & $\begin{array}{l}\text { Amazonas, Bolívar, Cesar, Cauca, Magdalena, Nariño, } \\
\text { Vaupés }\end{array}$ \\
\hline Medio (7) & $31.9-42.5$ & $\begin{array}{l}\text { Arauca, Caquetá, Casanare, Guaviare, Huila, Putumayo, } \\
\text { San Andrés, }\end{array}$ \\
\hline Bajo (7) & $21.8-31.8$ & $\begin{array}{l}\text { Antioquia, Atlántico, Boyacá, Meta, Norte de } \\
\text { Santander, Santander, Tolima }\end{array}$ \\
\hline Muy Bajo (6) & $9.2-21.7$ & $\begin{array}{l}\text { Bogotá, Caldas, Cundinamarca, Quindío, Risaralda, } \\
\text { Valle del Cauca }\end{array}$ \\
\hline
\end{tabular}

Fuente: elaboración propia a partir del índice de necesidades básicas insatisfechas a escala departamental, actualizado el 29 de junio del 2011; DANE

La información para los modelos de regresión binomial negativa se obtuvo a escala departamental para cada uno de los años analizados. La variable de respuesta de los modelos es la tasa de mortalidad estandarizada por suicidios calculada previamente. Las variables independientes socioeconómicas incluidas son: el índice de Gini, la incidencia de la pobreza, la tasa de desempleo, el año de ocurrencia, los grupos de edad y el sexo. Se excluyó de los modelos la variable de INB, debido a que solamente se tiene información para un año (2011), por lo que dicha variable no es consistente con el tipo de información utilizada. Se realizaron dos modelos de regresión: en el primero se incluyeron, únicamente, las características demográficas básicas de la población — sexo, grupo de edad y año de ocurrencia-; en el segundo modelo, además de las variables del modelo anterior, se agregaron las variables socioeconómicas.

40 Como se comentó previamente, se realizó un análisis de regresión múltiple, para lo cual se utilizó una regresión binomial negativa (RBN) de datos panel con efectos fijos en ambos modelos. Los modelos de RBN se utilizan cuando la variable de respuesta se considera de conteo y el supuesto de los modelos Poisson, de acuerdo con el cual la media es igual a la varianza, no se sostiene, lo que sucede a menudo cuando los datos presentan una gran dispersión (26), como en el caso de la tasa de mortalidad por suicidios. El modelo de regresión estimado es: $\ln \left(Y_{i}\right)=a_{i}+\beta n X n_{i t}$ $+y_{t}+u_{i t}$, donde $Y_{i t}$ es la tasa de suicidios para el departamento $i$ en el año $t$; $\mathrm{a}_{i}$ es el efecto fijo para el departamento $i$ que captura todos los factores no observables que no varían en el tiempo (2); ßn son los parámetros que se van a estimar; $X n_{i t}$ es el valor de cada covariable $n$ en el modelo (incluyendo los determinantes socioeconómicos) del departamento $i$ en el año $t ; \mathrm{y}_{t}$ es el efecto específico del tiempo; y $u_{i t}$ es el término del error aleatorio.

Se utilizó un modelo con efectos fijos, con base en los resultados obtenidos en la prueba 
de Hausman, ${ }^{2}$ en primer lugar (anexo 1), y segundo debido a que el termino fijo de la regresión puede controlar ciertas características no observadas de los departamentos que permanecen invariables en el tiempo, tales como cuestiones geográficas, históricas, culturales o socioeconómicas (2).

\section{Limitaciones}

Dado que en esta investigación la información sobre suicidios se obtuvo de las estadísticas vitales de mortalidad, es necesario tener en cuenta que, en general, existe cierta tendencia a ocultar los casos de suicidio por cuestiones culturales o religiosas, así como por problemas en los procedimientos de registro, por lo cual las defunciones por suicidios pueden estar subregistradas (27-28), y ello implicaría que las tasas de suicidios y su impacto en la esperanza de vida pudieran estar subestimados. A pesar de ello, la información de estadísticas vitales en Colombia se ha catalogado de buena y muy buena calidad (29).

Otra limitación del presente estudio es la imposibilidad de calcular la exposición a los factores de riesgo de aquellos individuos que cometieron un suicidio, debido a que la información socioeconómica solamente está disponible en un nivel agregado (2). De igual forma, como se comentó previamente, los datos utilizados en los modelos solo incluyen veinticuatro departamentos del país y no abarcan todo el periodo de estudio. Asimismo, debido a que los estudios ecológicos manejan datos agregados o grupales, por lo que no se pueden confirmar las asociaciones a un nivel individual (30-31), los resultados derivados de este tipo de investigaciones deben interpretarse con

2 La prueba de Hauman de especificación para modelos de efectos aleatorios es una prueba estadística que sirve para contestar a la pregunta sobre si se debe llevar a cabo un modelo de efectos fijos o aleatorios. Su definición e interpretación puede ser encontrada en Green (26). cautela. Sin embargo, dadas las características del fenómeno bajo estudio y sus factores asociados (como la desigualdad del ingreso, el porcentaje de población en pobreza y la tasa de desempleo), es necesario llevar a cabo un acercamiento ecológico que permita realizar un análisis tipo panel a escala nacional y departamental de la mortalidad por suicidio en Colombia (2).

\section{Resultados}

\section{Tasas de mortalidad}

Los suicidios representaron el $1.27 \%$ del total de muertes registradas en Colombia en el año 2000 y el $1.02 \%$ en el 2013. La razón hombre-mujer de las tasas osciló entre 3.3 y 4.6 suicidios masculinos por cada femenino, con una tendencia creciente. La tasa estandarizada de mortalidad por suicidios masculina disminuyó de 10 a 7.2 suicidios por 100000 habitantes entre el 2000 y el 2013 (un cambio de $28.1 \%$ ), mientras que para mujeres se redujo de 2.7 a 1.7 suicidios (cambio relativo de $36.9 \%$ ).

Por INB, las mayores tasas de mortalidad por suicidios masculinos en el 2000 se presentaron en el estrato medio, en tanto que las menores se dieron en el estrato muy alto (figura 1). Para el 2013, el estrato medio continuó siendo el estrato con mayor mortalidad por suicidios; sin embargo, tuvo una reducción importante de la incidencia de suicidios $(41.4 \%)$, junto con el estrato muy bajo (42.3\%). Los estratos alto y muy alto presentaron un incremento de la mortalidad por suicidios entre el $2000 \mathrm{y}$ el 2013 (1.6 y $3.9 \%$, respectivamente). En el caso de mujeres se presenta una situación similar, con las mayores tasas en el estrato medio y las más bajas en el estrato muy alto, pero, a diferencia de lo que sucede con los hombres, para mujeres todos los estratos del INB tuvieron una disminución de la mortalidad por suicidios, especialmente en los estratos medio y muy bajo. 


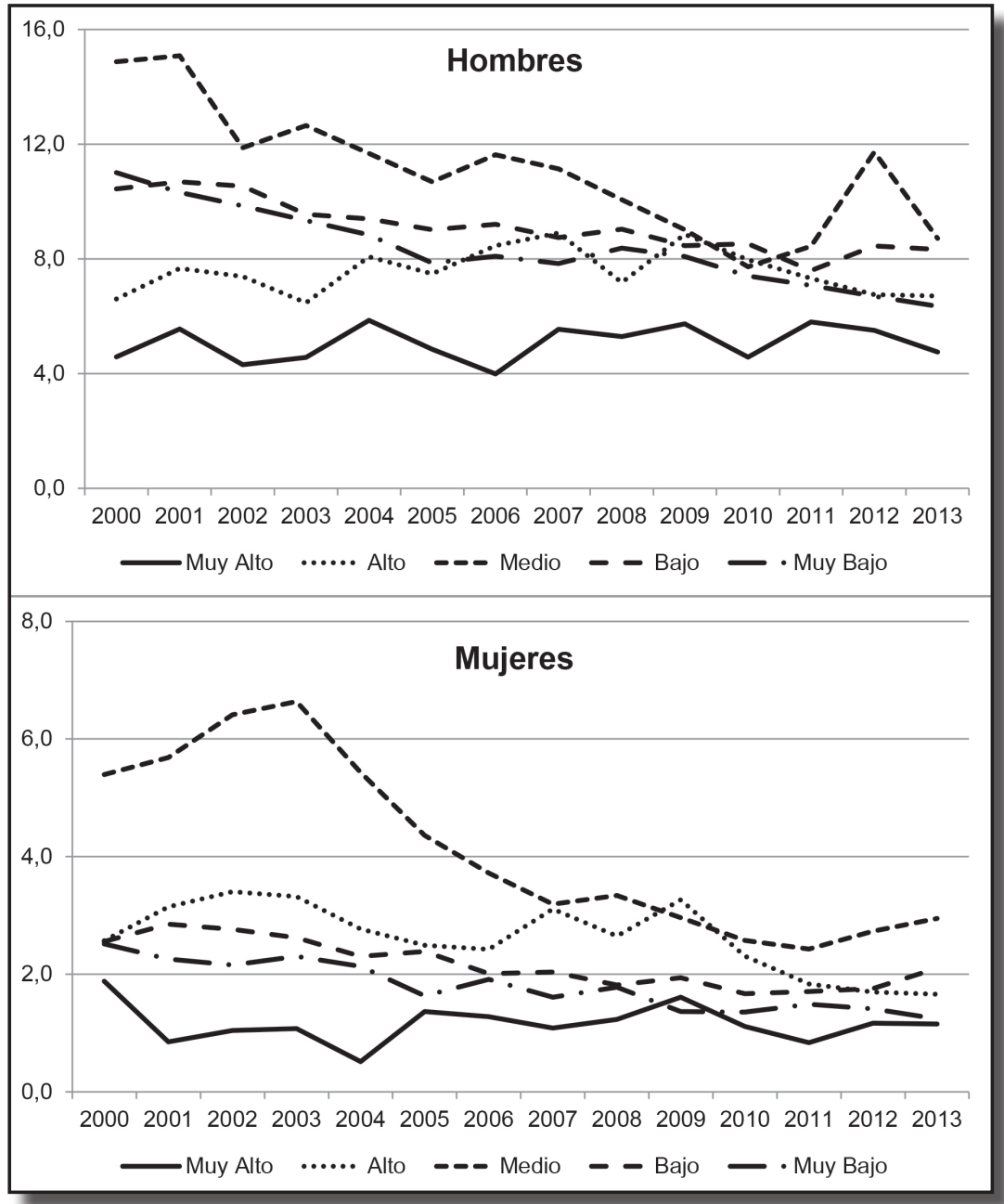

Figura 1. Tasas estandarizadas de mortalidad por Suicidios en los estratos del INB, Colombia 2000-2013

Fuente: elaboración propia con base en datos del DANE, 2000-2013

\section{Años de vida perdidos}

Los AVP por suicidios también presentaron una disminución a lo largo del periodo de estudio. Su impacto en la esperanza de vida temporaria entre los cero y los cien años fue de 0.32 años para hombres y 0.08 años para mujeres en el 2013; esto implica que, si se eliminaran las defunciones por suicidios en el 2013, por ejemplo, la esperanza de vida aumentaría en los niveles mencionados. Este descenso de los AVP por suicidios en hombres y mujeres se produjo en mayor medida entre los quince y los veintinueve años de edad, que es el grupo de edad en el cual los suicidios tienen un mayor impacto en la esperanza de vida de la población colombiana (tabla 2), se observó también un descenso importante de la mortalidad por suicidios entre los $30 \mathrm{y}$ los 49 años de edad en ambos sexos. 


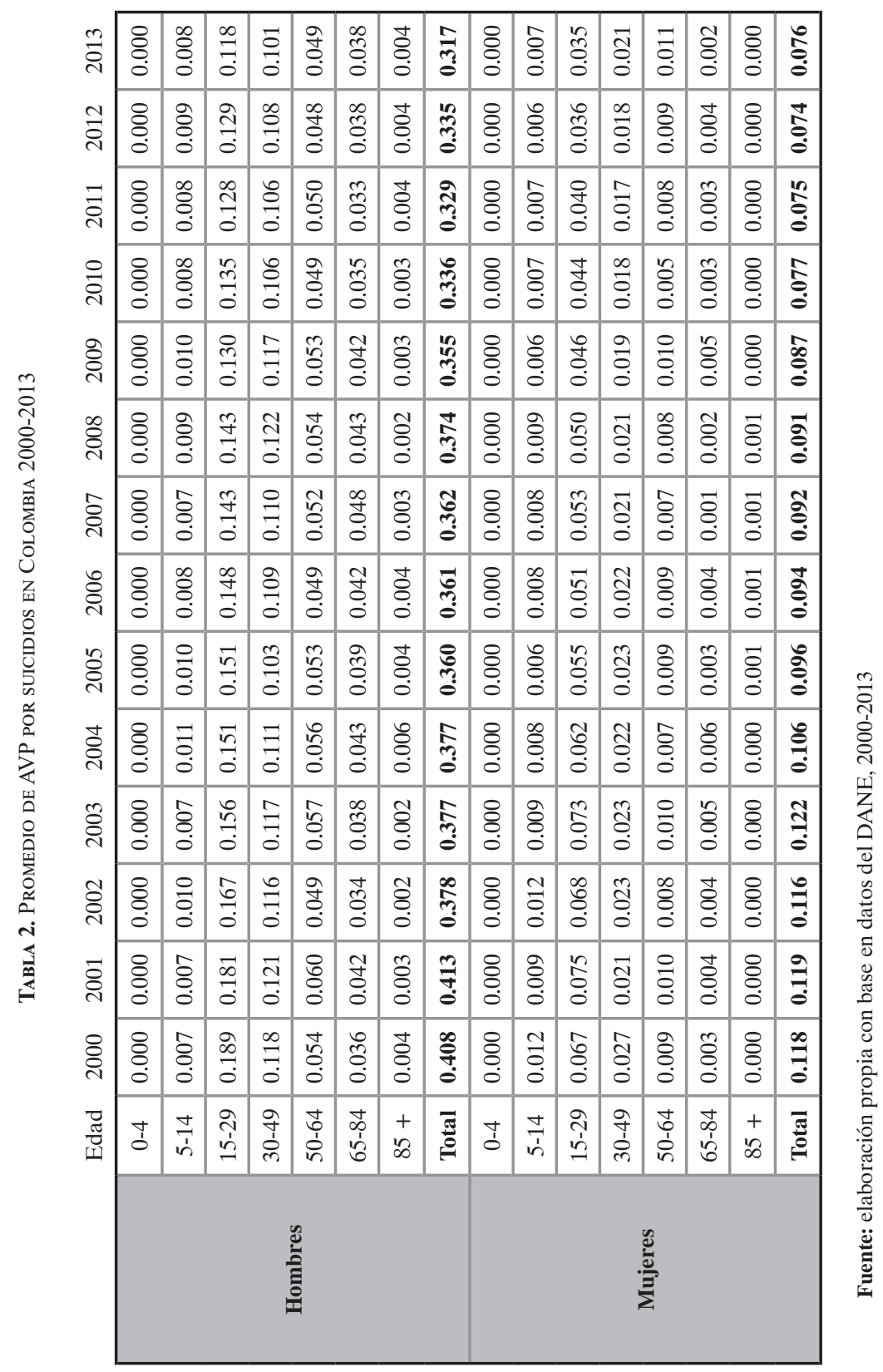

Por estrato de INB, se observa que en el 2000 el estrato medio tuvo los mayores AVP por suicidios masculinos (0.54 años), junto con el muy bajo (0.46 años); en el 2013 ambos estratos tuvieron la mayor disminución de la mortalidad por suicidios, de tal forma que el estrato muy bajo fue el que tuvo los menores AVP por suicidios en ese año (tabla 3). Para mujeres se observó una situación análoga, con el estrato medio como aquel con mayores niveles de AVP en el 2000, pero a diferencia de los hombres, dicho estrato se mantuvo como 
el que más AVP tuvo en el 2013; los estratos de muy bajo, muy alto y alto INB, fueron los que presentaron una mayor disminución del impacto de la mortalidad por suicidios femeninos entre el 2000 y el 2013. Destaca que todos los estratos presentaron una disminución de la mortalidad por esta causa en ambos sexos y se advierte cierta convergencia del valor de los AVP entre los estratos, especialmente en hombres.

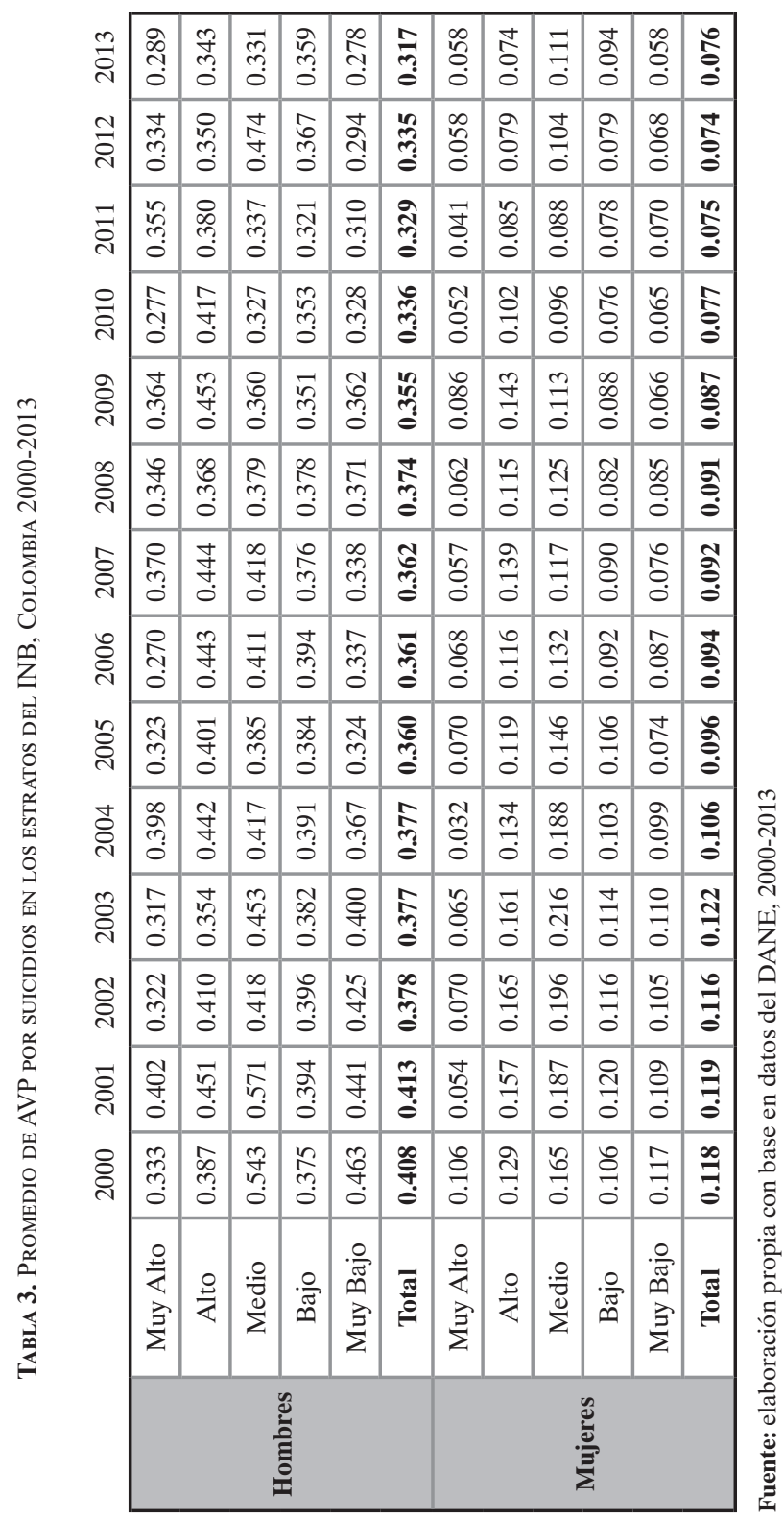




\section{Modelo de regresión binomial negativa}

La tabla 4 muestra los valores de las variables del índice de Gini, la incidencia de la pobreza y la tasa de desempleo. Se puede apreciar que la incidencia de la pobreza ha ido en descenso en Colombia en la última década. De igual forma, el índice de Gini ha mostrado una tendencia decreciente en el periodo $(0.572$ a 0.522), y la tasa de desempleo, al igual que los indicadores anteriores se ha reducido en los últimos años.

Tabla 4. Coeficientes de los modelos estimados para analizar la asociación entre la tasa de MORTALIDAD POR SUICIDIOS Y FACTORES SOCIOECONÓMICOS, COLOMBIA

\begin{tabular}{|c|c|c|c|c|c|}
\hline \multirow{2}{*}{ Variables } & \multirow{2}{*}{ Categorías } & \multicolumn{2}{|c|}{ Modelo 1} & \multicolumn{2}{|c|}{ Modelo 2} \\
\hline & & $\mathbf{R R}$ & IC $95 \%$ & $\mathbf{R R}$ & IC $95 \%$ \\
\hline \multirow{2}{*}{ Sexo } & Hombres + & 1.0000 & - & 1.0000 & - \\
\hline & Mujeres & 0.1278 & $(0.1142,0.1430)$ & 0.1245 & $(0.1111,0.1394)$ \\
\hline \multirow{15}{*}{ Edad } & $10-14$ & 2.2477 & $(1.5480,3.2638)$ & 2.1968 & $(1.5132,3.1892)$ \\
\hline & $15-19$ & 9.6132 & $(6.8449,13.501)$ & 9.5323 & $(6.7908,13.380)$ \\
\hline & $20-24$ & 6.6022 & $(4.9429,8.8185)$ & 6.4820 & $(4.8716,8.6247)$ \\
\hline & $25-29$ & 4.9380 & $(3.7267,6.5430)$ & 4.9250 & $(3.7253,6.5110)$ \\
\hline & $30-34$ & 3.6122 & $(2.7625,4.7231)$ & 3.7045 & $(2.8328,4.8445)$ \\
\hline & $35-39$ & 2.3928 & $(1.8263,3.1348)$ & 2.4629 & $(1.8774,3.2311)$ \\
\hline & $40-44$ & 1.8730 & $(1.4388,2.4383)$ & 1.9199 & $(1.4731,2.5020)$ \\
\hline & $45-49$ & 1.9534 & $(1.4976,2.5479)$ & 2.0512 & $(1.5684,2.6825)$ \\
\hline & $50-54$ & 1.8567 & $(1.4358,2.4009)$ & 1.9130 & $(1.4754,2.4804)$ \\
\hline & $55-59$ & 1.6352 & $(1.2641,2.1152)$ & 1.7426 & $(1.3414,2.2638)$ \\
\hline & $60-64$ & 1.1431 & $(0.8858,1.4752)$ & 1.1833 & $(0.9136,1.5325)$ \\
\hline & $65-69$ & 1.2947 & $(1.0025,1.6721)$ & 1.3272 & $(1.0237,1.7207)$ \\
\hline & $70-74$ & 1.1327 & $(0.8780,1.4614)$ & 1.1607 & $(0.8967,1.5024)$ \\
\hline & $75-79$ & 0.8896 & $(0.6839,1.1572)$ & 0.8849 & $(0.6781,1.1547)$ \\
\hline & 80 y más + & 1.0000 & - & 1.0000 & - \\
\hline \multicolumn{2}{|l|}{ Año } & 0.9588 & $(0.9549,0.9627)$ & 0.9449 & $(0.9389,0.9508)$ \\
\hline \multicolumn{2}{|c|}{ Tasa de desempleo } & - & - & 1.0374 & $(1.0300,1.0448)$ \\
\hline \multicolumn{2}{|c|}{ Incidencia de pobreza } & - & - & 0.9816 & $(0.9784,0.9848)$ \\
\hline \multicolumn{2}{|c|}{ Índice de Gini } & - & - & 2.8453 & $(1.2944,6.2542)$ \\
\hline \multicolumn{2}{|l|}{$\mathbf{N}$} & & 9022 & \multicolumn{2}{|c|}{902} \\
\hline
\end{tabular}

Fuente: elaboración propia con base en datos del DANE, 2000-2013

Como se observa en la tabla 4, el modelo de regresión con las variables demográficas muestra que las mujeres colombianas tuvieron un riesgo $87 \%$ menor que los hombres de fallecer por suicidio entre el 2000 y el 2013. Las edades que presentan un mayor riesgo de suicidio fueron de 15 a 39 años. Y, a medida que se incrementa la edad, disminuye el riesgo de fallecer por esta causa. Los años tuvieron una asociación negativa con la tasa de suicidios, lo que corrobora el descenso de la mortalidad por esta causa a lo largo del periodo de estudio. 
Estos resultados mantuvieron su significancia estadística en el modelo 2, el cual incluye las características socioeconómicas de los departamentos colombianos. Destaca que, a mayor tasa de desempleo, la tasa de suicidios se incrementa en un $3.7 \%$. A mayor incidencia de la pobreza, el riesgo de suicidio se reduce en $1.8 \%$. Y, finalmente, se observa que a mayor desigualdad del ingreso (medido por el coeficiente de Gini), el riesgo de suicidio se incrementa en casi tres veces (2.84).

\section{Discusión}

E1 suicidio es actualmente uno de los principales problemas de salud pública y representa una de las principales formas de violencia en Colombia y en el mundo (32). Se trata de un fenómeno multicausal, con una alta carga de mortalidad prematura y elevados costos sociales, económicos y psicológicos, ya que los intentos de suicidio tienen secuelas graves y, en ocasiones, permanentes en los afectados $(1,33)$.

En Colombia, la mortalidad por suicidios disminuyó de manera paulatina en el periodo del 2000 al 2013, tendencia que contrasta con el comportamiento del fenómeno a finales del siglo pasado, cuando la mortalidad por esta causa presentó un incremento significativo $(4,20)$. De igual forma, el impacto de este fenómeno en la esperanza de vida en Colombia se redujo de manera importante. Dicha caída se produjo, principalmente, entre los 15 y los 29 años de edad en ambos sexos. Por sexo se evidenció mayor mortalidad por suicidios en hombres que en mujeres, resultados que son similares a los encontrados en otras investigaciones relacionadas con el tema (32,34-36).

Por otro lado, dado que el INB se utiliza para 46 medir la magnitud de la pobreza en relación con la población (24), se analizó la posible relación del nivel y la tendencia de la mortalidad por suicidios con los distintos estratos de pobreza en el país. Dichos resultados muestran que el estrato medio tuvo el mayor impacto de la mortalidad por suicidios en Colombia entre el 2000 y el 2013, el estrato muy bajo presentó las mayores disminuciones de la incidencia de suicidios, en tanto que el estrato muy alto tuvo las menores tasas de mortalidad y, junto al estrato muy bajo, los menores AVP. Por tanto, fue el estrato donde los suicidios tuvieron el menor impacto en la esperanza de vida. Se observó, además, cierta convergencia de la mortalidad por suicidios entre los estratos, lo cual indica que, con independencia del nivel de pobreza, en Colombia se presentó una disminución de la mortalidad por suicidios a partir del 2000. Estos resultados parecen señalar que no existe una relación clara entre la pobreza, medida a través del INB, y la mortalidad por suicidios, por lo que dicha asociación se estudia más a profundidad por medio de los modelos de regresión.

En este sentido, se analizó qué relación existe entre la pobreza, la desigualdad, el desempleo y la mortalidad por suicidios, controlando por sexo, edad y año, a través de modelos de regresión binomial negativa. En el primer modelo se analizaron las características de la población y se observó que las mujeres tienen un menor riesgo de fallecer por suicidio que los hombres. Asimismo, el mayor riesgo de fallecer por suicidios se da en jóvenes y adultos jóvenes. Con el paso del tiempo, a medida que avanza el siglo XXI, en Colombia ha disminuido el riesgo de suicidio.

El segundo modelo muestra que la desigualdad del ingreso es un factor importante del suicidio en Colombia, ya que a medida que esta aumenta, el riesgo de fallecer por suicidio lo hace en casi tres veces. Esta investigación profundiza el estudio de la relación entre la desigualdad y la mortalidad 
por suicidio realizado en un trabajo previo en Colombia (37), trabajo que analiza esta asociación con una metodología puramente descriptiva, sin adentrarse en cómo median otras variables como el sexo, la edad, el año, la tasa de desempleo o la pobreza. De igual forma, otros estudios realizados en Brasil (2) y Japón (38-39) han investigado la relación entre la desigualdad del ingreso y las tasas de suicidio, y han encontrado que dicha asociación es positiva. Una posible explicación de esta relación es que la desigualdad del ingreso incrementa la fragmentación social (40), la cual, como postuló Durkheim, se convierte en un factor de riesgo del suicidio cuando las sociedades carecen de cohesión social y sentido de pertenencia (41).

De igual forma, se observó que al incrementarse la tasa de desempleo, también lo hace el riesgo de suicidio. Existen otros estudios previos que también encuentran que las tasas de suicidio y las tasas de desempleo tienen una relación positiva $(34,42-44)$; incluso, en algunos contextos se ha calculado que una persona desempleada tiene el doble de riesgo de cometer un suicidio que una persona empleada (45). Se ha argumentado que el desempleo puede afectar el riesgo de suicidio, a través de la reducción del ingreso, la pérdida de estabilidad y del bienestar económico de las personas, lo que deriva en un aumento de las deudas, desesperación y pérdida de autoestima (46-47).

En cambio, en esta investigación se observó que la pobreza tiene una relación negativa con la mortalidad de suicidios. Esta relación no es consistente en la literatura alrededor del tema, ya que en algunos trabajos (48-49) la pobreza se ha asociado positivamente con el riesgo de suicidio, mientras que en otros se ha encontrado que es el estatus socioeconómico bajo el que se relaciona con un mayor riesgo de suicidio $(34,42-43,50)$. En este sentido, el efecto que la precariedad económica y la pobreza tienen sobre el suicidio pudiera no presentarse de forma directa, sino a través de otros factores mediadores como el desempleo, el estrés financiero y la inestabilidad familiar (51-52). Sin embargo, al igual que en este estudio, en otras investigaciones se ha observado una relación negativa entre el porcentaje de la población en situación de pobreza y la tasa de suicidio (53-54), resultado que se puede relacionar con el argumento de que la violencia tiende a ocurrir en lugares con alta posibilidad de acumulación económica y con presencia débil del Estado, y no necesariamente en los sectores más pobres (54).

Como se puede observar, cada uno de los factores socioeconómicos estudiados en este trabajo presenta una asociación estadísticamente significativa con el riesgo de suicidio, pero su efecto se da de manera diferenciada. No se debe olvidar que el suicidio es un fenómeno multicausal que es resultado de una compleja interacción de factores, tanto del ambiente social y económico como de características individuales de la población (55-56). En este sentido, los principales factores de riesgo individuales asociados con una mayor incidencia de suicidio consumado son: un intento previo de suicidio, considerado el principal predictor del suicidio $(1,8)$; el sexo masculino, ya que se conoce ampliamente que los hombres tienen un mayor riesgo de fallecer por suicidio que las mujeres (57-61), aunque ellas intentan suicidarse con mayor frecuencia que los hombres, pero este es, finalmente concretado, más en ellos (62-67); los desórdenes mentales, principalmente la depresión (68-69) o aquellos causados por el abuso de sustancias, el desorden afectivo (unipolar y bipolar), el de personalidad (limítrofe y antisocial) y los desórdenes psicóticos (esquizofrenia) (70-71); el duelo y la sensación de pérdida (1); los bajos niveles de escolaridad $(2,30,72)$, que se relacionan con el suicidio de manera indirecta, ya que 
reflejan una deficiente condición social y económica de los individuos (73); altas tasas de divorcio, separación y menores tasas de matrimonio, que se han identificado como indicadores de fragmentación social y, por tanto, se asocian con un incremento del suicidio (74-79); el consumo de alcohol, el cual actúa como agente de desinhibición y fomenta la impulsividad, lo que incrementa la posibilidad del suicidio (80-81); el abuso de sustancias $(60,82)$ y las discapacidades o enfermedades terminales (1).

\section{Conclusiones}

Son necesarias acciones de prevención del suicidio que aborden tanto los factores de riesgo individuales como programas, planes y políticas orientados a disminuir la desigualdad en el acceso a los factores de acumulación de riqueza, en el acceso y la acumulación de capital humano, en el acceso a servicios y bienes públicos y, en general, en los estándares de vida (54). Se debe implementar, además, políticas de salud pública encaminadas a realizar acciones de identificación y atención oportuna y con una estrategia integral que incluya una mejor capacitación del personal de salud para el reconocimiento temprano y el tratamiento de calidad ante desórdenes psiquiátricos (83). Es necesario promover intervenciones en salud pública que disminuyan el acceso de las personas a métodos letales para suicidarse, como, por ejemplo, un mayor control de armas de fuego.

A través del documento se resaltó que, si bien el suicidio es un acto individual, se ve influido por, e impacta a, toda la sociedad. Se presentó evidencia de acuerdo con la cual, además de los factores individuales del suicidio, en Colombia las condiciones socioeconómicas del entorno deben ser consideradas factores de riesgo del suicidio
(2). Sin embargo, todavía se requiere mayor conocimiento de los mecanismos a través de los cuales los diversos factores de riesgo afectan la mortalidad por suicidio en el país y en diferentes contextos (84). Para ello se sugiere continuar con investigaciones que incorporen otros factores de riesgo del suicidio con diferentes metodologías, con el objetivo de proveer mayor conocimiento y comprensión del fenómeno que fomente la generación de políticas de prevención y atención oportuna, de tal manera que se pueda avanzar en el camino de la disminución del impacto de esta causa de muerte en la sociedad.

\section{Referencias bibliográficas}

1. Organización Mundial de la Salud (OMS). Prevención del suicidio un imperativo global. Washington D.C.: OMS; 2014.

2. Machado DB, Rasella D, dos Santos DN. Impact of income inequality and other social determinants on suicide rate in Brazil. PLoS ONE. 2015;10(4): $\mathrm{e} 0124934$.

3. Rendón-Quintero E, Rodríguez-Gómez R. Vivencias y experiencias de individuos con ideación e intento suicida. Rev Colomb Psiquiat. 2016;45(2):92-100.

4. Cendales R, Vanegas C, Fierro M, Córdoba R, Olarte A. Tendencias del suicidio en Colombia,1985-2002. Rev Panam Salud Publica. 2007;22(4):231-8.

5. Organización Mundial de la Salud (OMS). Informe mundial sobre la violencia y la salud. Ginebra: OMS; 2002.

6. Ying YH, Chang K. A study of suicide and socioeconomic factors. Suicide Life Threat Behav. 2009;39(2):214-26.

7. Organización Mundial de la Salud (OMS). El suicidio, un problema de salud pública enorme y sin embargo prevenible según la OMS [internet]. Ginebra: OMS; 2014 [acceso: 26 de agosto del 2016]. Disponible en: http://www.who.int/mediacentre/ news/releases/2004/pr61/es/

8. Hernández-Bringas HH, Flores-Arenales R. E1 suicidio en México. Papeles de Población. 2011;17(68):69-101.

9. Villalobos-Galvis F. Situación de la conducta suicida en estudiantes de colegios y universidades de San Juan de Pasto, Colombia. Salud Mental. 2009;32(2):165-71.

10. Makinen I. Are there social correlates to suicide? Soc Sci Med. 1997;44(12):1919-29.

11. Hawton K, Harriss L, Hodder K, Simkin S, Gunnell D. The influence of the economic and social environment on deliberate self-harm and suicide: an ecological and person-based study. Psychol Med. 2001;31(5):827-36. 
12. Page A, Morrell S, Taylor R. Suicide differentials in Australian males and females by various measures of socio-economic status, 1994-98. Aust N Z J Public Health. 2002; 26(4):318-24.

13. Qin P, Agerbo E, Mortensen PB. Suicide risk in relation to socioeconomic, demographic, psychiatric, and familial factors: a national register-based study of all suicides in Denmark, 1981-1997. Am J Psychiatry. 2003;160(4):765-72.

14. Stack S. Suicide: a 15-year review of the sociological literature. Part I: cultural and economic factors. Suicide Life Threat Behav. 2000; 30(2):145-61.

15. Stuckler D, Basu S, Suhrcke M, Coutts A, McKee M. The public health effect of economic crises and alternative policy responses in Europe: an empirical analysis. Lancet. 2009; 374(9686):315-23.

16. Uutela A. Economic crisis and mental health. Curr Opin Psychiatry. 2010;23(2):127-30.

17. Organización Panamericana de la Salud (OPS). Mortalidad por suicidio en las Américas. Washington D.C.: OPS; 2014.

18. Moyano E, Barría R. Suicidio y producto interno bruto en Chile: hacia un modelo predictivo. Rev Lat Am Psicol. 2006; 38(2): 343-359.

19. Rodríguez MA, Díaz LM, Hernández MA, Tobón JA. Mortalidad por suicidio en Colombia. Medicina UPB. 2006; 25(2):159-70.

20. Gómez-Restrepo C, Rodríguez N, de Romero L, Pinilla C, López E, Díaz-Granados N, et al. Suicidio y lesiones autoinfligidas, Colombia, 1973-1996. Rev Colomb Psiquiatr. 2002;31(2):123-36.

21. Departamento Administrativo Nacional de Estadística (DANE). Pobreza y desigualdad [internet]. Bogotá: DANE; 2015 [acceso: 26 de agosto del 2016]. Disponible en http://www.dane.gov.co/index.php/estadisticas-por-tema/pobreza-y-condiciones-de-vida/ pobreza-y-desigualdad

22. Arriaga E. Los años de vida perdidos: su utilización para medir el nivel y cambio de la mortalidad. Notas de Población. 1996; 24(63):7-38.

23. Bocco M. La relación entre los años de vida perdidos y la esperanza de vida: aplicaciones para el análisis de la mortalidad. Notas de Población. 1996; 24(63):39-60.

24. García P. Indicadores sociales departamentales. En: Departamento Nacional de Planeación, editor. Colombia: Boletines Sistema de Indicadores Sociodemográficos; 2007.

25. Fresneda O. La medida de necesidades básicas insatisfechas (NBI) como instrumento de medición de la pobreza y focalización de programas. Serie Estudios y Perspectivas (Cepal). 2007;18:1-193.

26. Greene W. Econometric analysis. New Jersey: Prentice Hall; 2011.

27. Cifuentes S. Comportamiento del suicidio, Colombia, 2013. En: Instituto Nacional de Medicina Legal y Ciencias Forenses. Forensis 2013, Datos para la vida. Bogotá: Colombia; 2014. p.127-68.

28. Moreno J, Sánchez R. Muertes por causas violentas y ciclo económico en Bogotá, Colombia: un estudio de series de tiempo, 1997-2006. Rev Panam Salud Publica. 2009;26(1):23-30.
29. Palacio C, García J, Diago J, Zapata C, López G, Ortiz J, et al. Identification of suicide risk factors in Medellín, Colombia: a case-control study of psychological autopsy in a developing country. Arch Suicide Res. 2007;11(3):297-308.

30. González A, Rodríguez A, Aristizábal A, García J, Palacio C, López C. Suicidio y género en Antioquia (Colombia): estudio de autopsia psicológica. Rev Colomb Psiquiat. 2010;39(2):251-67.

31. Rodríguez-Escobar JA, Medina-Pérez OA, Cardona-Duque DV. Caracterización del suicidio en el departamento de Risaralda, Colombia, 2005-2010. Rev Fac Med. 2013;61(1):9-16.

32. Campo-Arias A y Herazo E. Asociación entre desigualdad y tasa de suicidio en Colombia (1994-2013). Rev Colomb Psiquiat. 2015; 44(1):28-32.

33. Chen J, Choi YJ, Sawada Y. How is suicide different in Japan? Japan and the World Economy. 2009;21(2):140-50.

34. Kazuyuki I. Income inequality and the suicide rate in Japan: Evidence from cointegration and LA-VAR. J Appl Econ. 2010; 13(1):113-33.

35. Rehkopf DH, Buka SL. The association between suicide and the socio-economic characteristics of geo-graphical areas: a systematic review. Psychol Med. 2006;36(2):145-57.

36. Jiménez-Ornellas RA, Cardiel-Téllez L. El suicidio y su tendencia social en México: 1990-2011. Papeles de Población. 2013;19(77):205-29.

37. Cavanagh JT, Carson AJ, Sharpe M, Lawrie SM. Psychological autopsy studies of suicide: A systematic review. Psychol Med. 2003;33(3):395-405.

38. Sánchez R, Orejarena S, Guzmán Y, Forero J. Suicidio en Bogotá: un fenómeno que aumenta en poblaciones jóvenes. Biomédica. 2002;22:417-24.

39. Norstrom T, Gronqvist H. The Great Recession, unemployment and suicide. J Epidemiol Community Health. 2015;69(2):110-6.

40. Kposowa AJ. Unemployment and suicide: a cohort analysis of social factors predicting suicide in the US National Longitudinal Mortality Study. Psycho Med.2001;31(1):127-38.

41. Platt S. Unemployment and suicidal behavior: A review of the literature. Soc Sci Med. 1984;19(2):93115.

42. Stack S, Haas A. The effect of unemployment duration on national suicide rates: a time series analysis, 1948-1982. Sociol Focus. 1984;17(1):17-29.

43. Inoue K, Fujita Y, Takeshita H, Abe S, Fujihara J, Ezoe S, Sampei M, Miyaoka T, Horiguchi J, Okazaki Y, Fukunaga T. A Long-term Study of the Association between the Relative Poverty Rate and Suicide Rate in Japan. J Forensic Sci. 2016;61:S140-3.

44. Bernburg JG, Thorlindsson T, Sigfusdottir ID. The spreading of suicidal behavior: The contextual effect of community household poverty on adolescent suicidal behavior and the mediating role of suicide suggestion. Soc Sci Med. 2009;68(2):380-9.

45. Schony W, Grausgraber A. Epidemiological data on suicide in upper Austria. Crisis. 1987;8(1):49-52.

46. Stack S. Suicide: A decade review of the sociological literature. Deviant Behavior. 1982;4(1):41-66. 
47. Nazareth S, Gomes C, Müller N, Faria X, Carvalho A De, Werner J. Características epidemiológicas do suicídio no Rio Grande do Sul. Rev Saúde Pública. 2004;38(6):804-10.

48. Campo-Arias A, Herazo E. Pobreza, desigualdad y tasa de suicidio en Colombia, 2012. Revista Duazary. 2014;11(2):126-30.

49. Xavier N, Gomes C, Nazareth S, Alves L, Werner J. Suicide rates in the State of Rio Grande do Sul, Brazil: association with socioeconomic, cultural, and agricultural factors. Cad Saúde Pública. 2006;22(12):2611-21.

50. Sarmiento A, Becerra LM. Análisis de las relaciones entre violencia y equidad [internet]. Bogotá: Departamento Nacional de Planeación; 1998 [acceso: 27 de agosto del 2016]. Disponible en: https://colaboracion.dnp.gov.co/CDT/Estudios \%20 Econmicos/93.pdf

51. Ortega PA, Manrique RD, Tovilla CA, López C, Cuartas JM. Clinical and epidemiological characteristics of suicides committed in Medellin, Colombia. Rev Colomb Psiquiat. 2014; 43(2):106-12.

52. Organización Mundial de la Salud (OMS). Public health action for the prevention of suicide. A framework. Ginebra: OMS; 2012.

53. Quinlan-Davidson M, Sanhueza A, Espinosa I, Escamilla-Cejudo JA, Maddaleno M. Suicide among young people in the Americas. J of Adolesc Health. 2014;54(3):262-8.

54. Joiner TE. Why people die by suicide. Cambridge: Harvard University Press; 2005.

55. Canetto SS. Women and suicidal behavior: A cultural analysis. Am J Orthopsychiatry. 2008;78(2):259-66.

56. Coleman D. Traditional masculinity as a risk factor for suicidal ideation: cross-sectional and prospective evidence from a study of young adults. Arch Suicide Res. 2015;19(3):366-84.

57. Coleman D, Kaplan MS, Casey JT. The social nature of male suicide: a new analytic model. Int J Mens Health. 2011;10(3):240-52.

58. Pietro D, Tavares M. Risk factors for suicide and suicide attempt: incidence, stressful events and mental disorders. J Bras Psiquitr. 2005; 54(2):146-54.

59. Runeson B, Tidemalm D, Dahlin M, Lichtenstein P, Långström N. Method of attempted suicide as predictor of subsequent successful suicide: national long term cohort study. BMJ. 2010;340:c3222.

60. Canetto SS, Sakinofsky I. The gender paradox in suicide. Suicide Life Threat Behav. 1998;28(1):1-23.

61. Organización Mundial de la Salud (OMS). Figures and facts about suicide. Ginebra: OMS; 1999.

62. Organización Mundial de la Salud (OMS). Mental health and development targeting people with mental health condition as a vulnerable group. Ginebra: OMS; 2010.

63. Stefanello S, da Silva Cais CF, Mauro MLF, et al. Gender differentiation in suicide attempts: preliminary results of the multisite intervention study on suicidal behavior (SUPRE-MISS) from Campinos, Brasil. Rev Bras Psiquiatr. 2008;30(2):139-43.

64. Borges G, Angst J, Nock MK, Ruscio AM, Kessler RC. Risk factors for the incidence and persistence of suicide-related outcomes: A 10-year follow-up study using the National Comorbidity Surveys. J Affect Disord. 2008;105(1-3):25-33.

65. Harris EC, Barraclough B. Suicide as an outcome for mental disorders. A meta-analysis. Br J Psychiatry. 1997;170:205-28.

66. Arsenault-Lapierre G, Kim C, Turecki G., 2004. Psychiatric diagnoses in 3275 suicides: a meta-analysis. BMC Psychiatry. 2004;4:37.

67. Hawton K, van Heeringen K. Suicide. The Lancet. 2009;373(9672):1372-81.

68. Posada-Villa J, Camacho JC, Valenzuela JI, Arguello A, Cendales JG, Fajardo R. Prevalence of suicide risk factors and suicide-related outcomes in the National Mental Health Study, Colombia. Suicide Life Threat Behav. 2009;39(4):408-24.

69. Manrique RD, Ochoa FL, Fernández LM, Escamilla R, Vélez E. Comportamiento del suicidio en Antioquia 1998-2000. Revista Ces Medicina. 2002;16(3):7-17.

70. Faria NM, Facchini LA, Fassa AG, Tomasi E. Estudo transversal sobre saúde mental de agricultores da Serra Gaúcha (Brasil). Rev Saúde Pública. 1999;33(4):391-400.

71. Agerbo E, Nordentoft M, Mortensen PB. Familial, psychiatric, and socioeconomic risk factors for suicide in young people: nested case-control study. BMJ. 2002;325(7355):74.

72. Pickett W, King WD, Lees RE, Bienefeld M, Morrison HI, Brison RJ. Suicide mortality and pesticide use among Canadian farmers. Am J Ind Med. 1998;34(4):364-72.

73. Marin-Leon L, Barros MB. Óbitos por suicídio: diferenças de gênero e nível socioeconômico. Rev Saúde Pública. 2003;37(3):357-63.

74. Gunnell D, Middleton N, Whitley E, Dorling D, Frankel S. Why are suicide rates rising in young men but falling in the elderly? A time-series analysis of trends in England and Wales 1950-1998. Soc Sci Med. 2003;57(4):595-611.

75. Aihara H, Iki M. Effects of socioeconomic factors on suicide from 1980 through 1999 in Osaka Prefecture, Japan. J Epidemiol. 2002;12(6):439-49.

76. Kendall R. Alcohol and suicide. Subst Alcohol Actions Misuse. 1983;4(2-3):121-7.

77. Skog O. Alcohol and suicide - Durkheim revisited. Acta Sociologica. 1991;34(3):193-206.

78. Harris EC, Barraclough BM. Suicide as an outcome for medical disorders. Medicine (Baltimore). 1994;73(6):281-96.

79. Rodríguez A. El suicidio y su prevención. En: Instituto Nacional de Medicina Legaly Ciencias Forenses (Forensis). Datos para la vida. Bogotá: Forensis; 2006. p. 275-316.

80. Madge N, Harvey J. Suicide among the young the size of the problem. J Adolesc. 1999;22(1):145-55.

81. Mahapatra P, Shibuya K, Lopez AD, Coullare F, Notzon $\mathrm{FC}$, Rao C, et al. Civil registration systems and vital statistics: successes and missed opportunities. Lancet. 2007;370(9599):1653-63.

82. Wake】eld J. Ecologic studies revisited. Annu Rev Public Health. 2008; 29:75-90.

83. Borges G, Orozco R, Benjet C, Medina-Mora ME. Suicidio y conductas suicidas en México: retros- 
pectiva y situación actual. Salud Publica Mex. 2010; 52(4):292-304.

84. Burrows S, Laflamme L. Socioeconomic disparities and attempted suicide: state of knowledge and implications for research and prevention. Int J Inj Contr Saf Promot. 2010;17(1):23-40.

\section{Anexo 1. Resultados de prueba} de Hausman de efectos fijos vs. efectos aleatorios

---- Coeficientes----

(b) (B) (b-B) sqrt (diag $\left.\left(\mathrm{V}_{-} b-\mathrm{V}_{-} \mathrm{B}\right)\right)$

Diferencia E.E. sexo -2.083605 -1.888169-.1954358.0315989

4bn.edad80 .787003 -.133253.9202557 .1253154

5.edad80 2.254682 1.407677.8470052.1062400

6.edad80 1.869029 1.346237.5227920.0716574

7.edad80 1.594318 1.129858.4644604.0666239

8.edad80 1.309561.913175.3963859.0596336

9.edad80 901351.557799.3435524.0605791

10.edad80.652254.369767.2824863.0569615

11.edad80.718401.436927.2814743.0583094

12.edad80.648688.411280.2374079.0540816

13.edad80.555388.367063.1883247.0546288

14.edad80.168281.062520.1057607.0532001

15.edad80.283054.175928.1071252.0534126 16.edad80.148989.092370.0566187.0532024

17.edad80 -.122291 -.154458.0321666 .0557326

año $-.056729-.047577-.0091511$.

tasadesempño .036686.035171.0015148 .

pobreza -.018573-.012326-.0062463.0004794

gini 1.045666 258919.7867462 .

$\mathrm{b}=$ consistent under Ho and Ha; obtained from xtnbreg

$\mathrm{B}=$ inconsistent under $\mathrm{Ha}$, efficient under Ho; obtained from xtnbreg

Test: Ho: difference in coefficients not systematic

$\operatorname{chi} 2(19)=(b-B){ }^{\prime}\left[\left(V_{-} b-V_{-} B\right)^{\wedge}(-1)\right](b-B)$

$=587.72$

Prob $>$ chi $2=0.0000$

( $\mathrm{V}_{-} \mathrm{b}-\mathrm{V} \_\mathrm{B}$ is not positive definite)

Estos resultados indican que se rechaza la hipótesis de que los efectos individuales sobre la tasa de mortalidad por suicidios se modelan de manera correcta por medio de una regresión con efectos aleatorios. Por tanto, se debe utilizar un modelo de efectos fijos para estudiar la asociación entre las variables socioeconómicas y la mortalidad por suicidios. 